\title{
Synthesis of nanosized $\mathrm{TiO}_{2}$ powders via a hydrothermal microemulsion process
}

\author{
Chung-Hsin Lu*, Ming-Chang Wen \\ Electronic and Electro-optical Ceramics Laboratory, Department of Chemical Engineering, National Taiwan University, Taipei, Taiwan, ROC
}

Received 24 October 2006; received in revised form 23 December 2006; accepted 1 January 2007

Available online 16 January 2007

\begin{abstract}
A microemulsion process combined with hydrothermal techniques has been developed for synthesizing anatase-type titania in this study. Nanosized $\mathrm{TiO}_{2}$ powders were successfully prepared by employing this hydrothermal microemulsion process. The particle and crystallite sizes of the formed $\mathrm{TiO}_{2}$ powders significantly depended on the $\mathrm{pH}$ value of microemulsion solutions. Low $\mathrm{pH}$ microemulsion solutions resulted in the formation of spherical particles, whereas neutral solutions gave rise to rod-like particles. The mechanisms of nucleation and crystal growth were influenced by the acidity of the solutions. The rod-like particles had much larger specific surface area than the spherical ones. The large surface area of the rod-like particles resulted in significantly enhanced photocatalytic activity.
\end{abstract}

(C) 2007 Elsevier B.V. All rights reserved.

Keywords: Microemulsion; Hydrothermal; Photocatalysis; Nanoparticles; Titania

\section{Introduction}

The impact of morphology on the properties of materials having large surface area is a field of increasing importance for understanding, creating and improving materials for diverse applications. It has been reported that the efficiency of photocatalytic reactions is markedly affected by one or more morphological parameters of the catalyst [1]. Apparently, morphological control is beneficial for obtaining materials with desirable surface area. Therefore, to synthesize catalysts with controlled morphology is of great technological interest. $\mathrm{TiO}_{2}$ is one of the most important catalytic materials. Literature reports reveal various approaches for the synthesis of nano-particles of titania, including the thermal hydrolysis [2-4], the sol-gel [5-8], the hydrothermal processes [9-11], and the microemulsion processes [12-16]. The powders produced by thermal hydrolysis process have serious problems of agglomeration. The disadvantage of the powders produced by sol-gel method is the long aging time for preparing the gel of powders and also not very economi$\mathrm{cal}$. The following calcination process of thermal hydrolysis and sol-gel methods would cause grain growth and agglomeration

\footnotetext{
* Corresponding author. Tel.: +88622365 1428; fax: +886223623040.

E-mail address: chlu@ntu.edu.tw (C.-H. Lu).
}

of the nano-particles. These two routes will inevitably lead to coarsening of the calcined powders and reduce the active surface area for photocatalysis.

The microemulsion process has the advantage of obtaining nanosized particles without serious agglomeration. The microemulsion system is a thermodynamically stable and optically isotropic solution formed by combining two immiscible liquids [17]. In order to prevent grain growth of the nanoparticles, a new hydrothermal microemulsion process has been developed in this study. Aqueous micelles in microemulsions were used as microreactors to confine the growth of $\mathrm{TiO}_{2}$ powders. Hydrothermal treatment was carried out at a relatively low crystallization temperature to prevent agglomeration of the asprepared particles [18]. The effects of $\mathrm{pH}$, temperature, reaction conditions on controlling the morphology and formation of the particles are described in this paper. The influence of the preparation conditions on the photocatalytic activity of $\mathrm{TiO}_{2}$ powders is also discussed.

\section{Experimental}

In the microemulsion system, cyclohexane was used as the continuous oil phase, $n$-hexanol as the cosurfactant, and OP-10 as the surfactant. The dispersed aqueous phase was composed of $\mathrm{TiOCl}_{2}$ solution and $1.0 \mathrm{M} \mathrm{NaOH}$ solution. The concentration of $\mathrm{TiOCl}_{2}$ solution was $0.2 \mathrm{M}$. In the continuous oil phase of the microemulsion system, the ratio of $n$-hexanol:polyoxyethylene (10) octylphenyl 
ether (OP-10):cyclohexane was set to be 3:2:10. Microemulsion I was prepared by mixing $100 \mathrm{ml}$ of the continuous oil phase and $10 \mathrm{ml}$ of the $\mathrm{TiOCl}_{2}$ aqueous solution to form the dispersed aqueous phase. In microemulsion II, $10 \mathrm{ml}$ of $1.0 \mathrm{M} \mathrm{NaOH}$ aqueous solution was mixed with $100 \mathrm{ml}$ of the continuous oil phase. The water-to-oil ratios in microemulsions I and II were both fixed at 1/10. The dispersed aqueous phase of microemulsion II was a precipitating reagent. The two microemulsion solutions were then mixed with vigorous stirring at room temperature for $30 \mathrm{~min}$. After the stirring process, the $\mathrm{pH}$ value of the mixed microemulsion was measured. The $\mathrm{pH}$ value of the mixed microemulsion was adjusted by varying the volume ratio of microemulsion I to microemulsion II. A colloidal solution was formed after stirring. The mixed microemulsion was placed in a Teflon container and treated hydrothermally at $150^{\circ} \mathrm{C}$ for $1 \mathrm{~h}$. The heating rate of autoclave was $3{ }^{\circ} \mathrm{C} / \mathrm{min}$. White suspension was obtained after the hydrothermal treatment. The suspension in the container was washed with ethanol and deionized water repeatedly to remove any residual organic species, and with acetone to prevent agglomeration among the particles. The washed suspension was then filtrated by vacuum filtration. The white powders obtained after filtration was dried at $60^{\circ} \mathrm{C}$ for $6 \mathrm{~h}$ in a vacuum drying oven.

The crystal phase of the as-prepared powders was identified via powder $\mathrm{X}$-ray diffraction (XRD) using $\mathrm{Cu} \mathrm{K} \alpha$ radiation at room temperature. The crystallite size of the powders was calculated from the Debye-Scherrer's equation. The morphology and primary particle size were observed via transmission electron microscopy (TEM). Specific surface area of the synthesized powders was determined by using the Brunauer-Emmett-Teller (BET) nitrogen adsorption method. The photocatalytic activities of the prepared samples were evaluated based on the decomposition of methylene blue in the aqueous solution. For the absorption of solution to satisfy the restrictions of Bill's law, the starting concentration of methylene blue solution was set at $30 \mu \mathrm{M}$. $0.01 \mathrm{~g}$ of the as-prepared powders were dispersed in $20 \mathrm{ml}$ of $30 \mu \mathrm{M}$ methylene blue solution. The solutions were irradiated at $365 \mathrm{~nm}$ for $90 \mathrm{~min}$ with an UV-lamp. The suspensions were centrifuged for pre-set durations to separate out the catalyst from the solution. The absorbance of the characteristic peak of methylene blue $(664 \mathrm{~nm})$ was measured using an UV-vis spectrometer.

\section{Results and discussion}

\subsection{Formation of nanosized $\mathrm{TiO}_{2}$ powders via the hydrothermal microemulsion process}

The mixed microemulsions were heated in a hydrothermal container at $150{ }^{\circ} \mathrm{C}$ for $1 \mathrm{~h}$. The $\mathrm{pH}$ values of the mixed microemulsions were varied in advance. The XRD patterns of the obtained powders synthesized at different $\mathrm{pH}$ values are illustrated in Fig. 1. Well-crystallized powders were obtained after the hydrothermal treatment. All of the samples showed diffraction peaks well consistent with the data listed in ICDD no. 21-1272 [19], indicating that they were anatase-type titania. The crystallite sizes of $\mathrm{TiO}_{2}$ powders were calculated from the XRD patterns. The crystal size of the prepared $\mathrm{TiO}_{2}$ powders increased with increasing $\mathrm{pH}$ value. The crystal size of $\mathrm{TiO}_{2}$ powders was $6 \mathrm{~nm}$ at $\mathrm{pH} 4$. When the $\mathrm{pH}$ value was increased to 7 , the crystallite size enlarged to $17 \mathrm{~nm}$. In addition, the diffraction peak (101) of the prepared powders became sharpened. As seen in Fig. 2 later, the change in the width of the diffraction peaks is related to the variation in microstructures of the obtained powders.

The microstructures of the obtained $\mathrm{TiO}_{2}$ powders are shown in Fig. 2. Fig. 2(a)-(c) shows the TEM photographs of the samples prepared from the starting microemulsion solutions with $\mathrm{pH} 4-6$, respectively. All the samples exhibited a uniform and spherical morphology. The powders prepared at $\mathrm{pH} 4-6$ of the starting microemulsion solutions have average particle sizes of

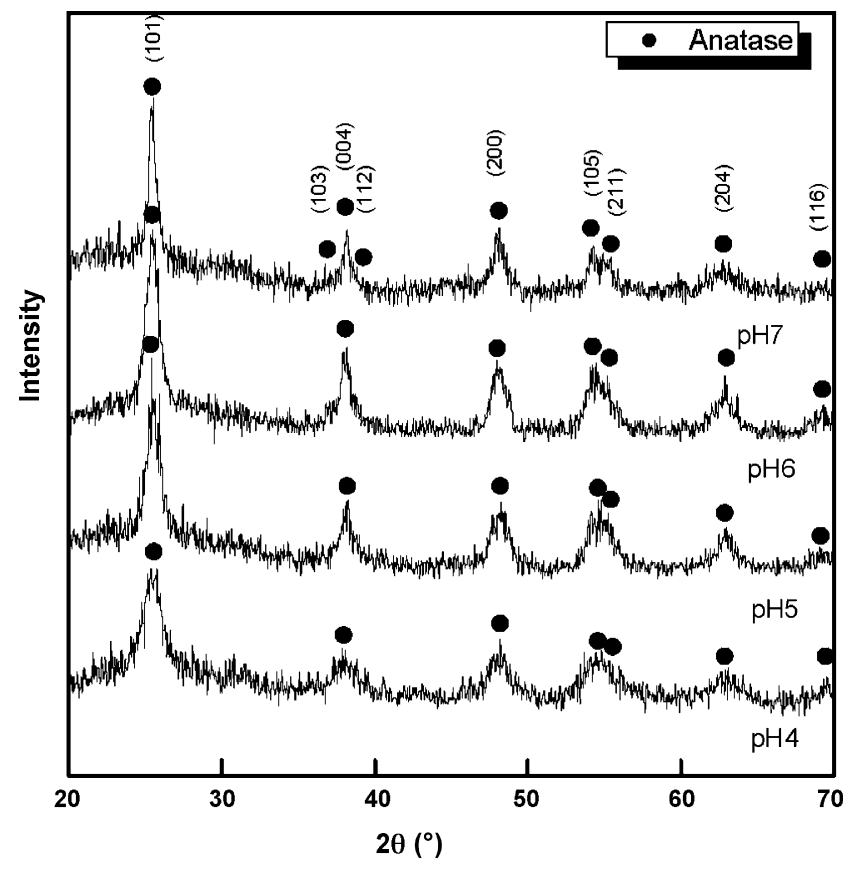

Fig. 1. X-ray diffraction patterns of $\mathrm{TiO}_{2}$ powders prepared via the hydrotherma microemulsion process.

around 10,15 and $20 \mathrm{~nm}$, respectively. It is noted that the particle size of $\mathrm{TiO}_{2}$ powders increases with an increase in the $\mathrm{pH}$ value of the microemulsion solution. On the other hand, when the $\mathrm{pH}$ value was increased to 7 , the formed particles had a specific rod-like morphology. As shown in Fig. 2(d) and (e) (the magnified TEM micrograph), the prepared powders have a large aspect ratio. This kind of morphology is considered to facilitate the crystal growth along a specific direction, thereby resulting in an increase in the crystal size.

Fig. 3 illustrates the mechanisms of the formation of the spherical and rode-like particles prepared from the starting microemulsion solution at $\mathrm{pH} \mathrm{4-7.} \mathrm{The} \mathrm{crystal} \mathrm{structure} \mathrm{of}$ anatase-type titania was considered to be the arrangement of $\mathrm{TiO}_{6}$ octahedra $[20,21]$ which would affect the morphology of the particles. The arrangement of $\mathrm{TiO}_{6}$ octahedra in anatase-type titania was edge shared in all directions [22]. In this study, the $\mathrm{pH}$ of the starting microemulsion solution would influence the morphology and particle size of the formed powders. The $\mathrm{pH}$ of the solution determines the concentration of the $\mathrm{OH}^{-}$groups which would affect the number of $\mathrm{OH}^{-}$groups joined with the $\mathrm{Ti}^{4+}$ complex center. The other positions connected with $\mathrm{Ti}^{4+}$ complex center would be occupied by chloride ions. It was suggested that the $\mathrm{Ti}^{4+}$ complex ion has the formula $\left[\mathrm{Ti}(\mathrm{OH})_{n} \mathrm{Cl}_{m}\right]^{2-}$ during the starting hydrolysis process [9].

When the acidity of the starting solution was high, the number of $\mathrm{OH}^{-}$groups in $\left[\mathrm{Ti}(\mathrm{OH})_{n} \mathrm{Cl}_{m}\right]^{2-}$ was less. The number of $\mathrm{OH}^{-}$ groups joined with $\mathrm{Ti}^{4+}$ complex center is considered to be 2-3 in acidified starting microemulsion solution as shown in Fig. 3 [9]. The two $\mathrm{OH}^{-}$groups joined with different $\mathrm{Ti}^{4+}$ complex centers can easily combine with each other to form a Ti-O-Ti bridge bond, and a water molecule would be eliminated through the above dehydration process. The dehydration of $\mathrm{OH}^{-}$groups 


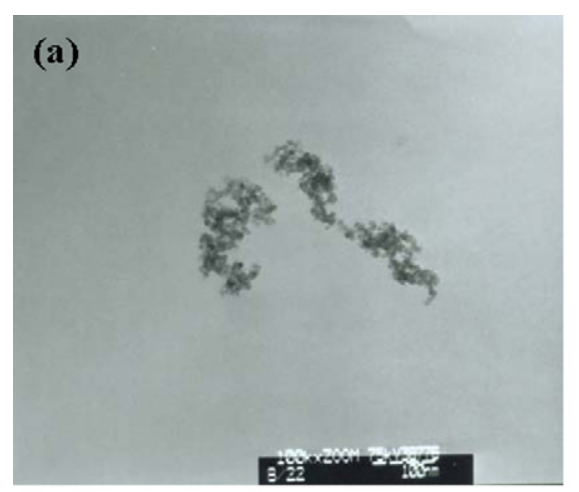

$\mathrm{pH} 4$

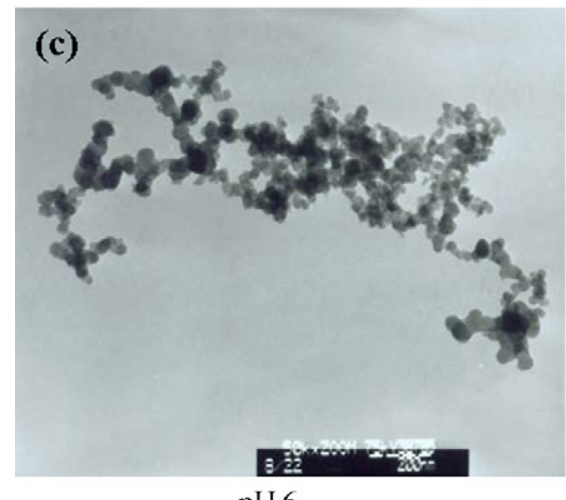

$\mathrm{pH} 6$
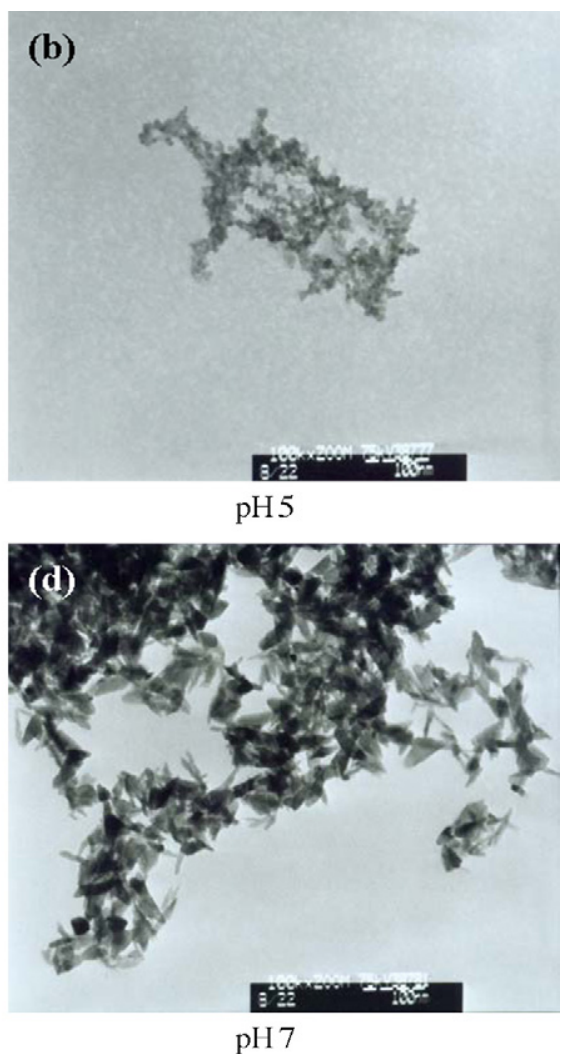

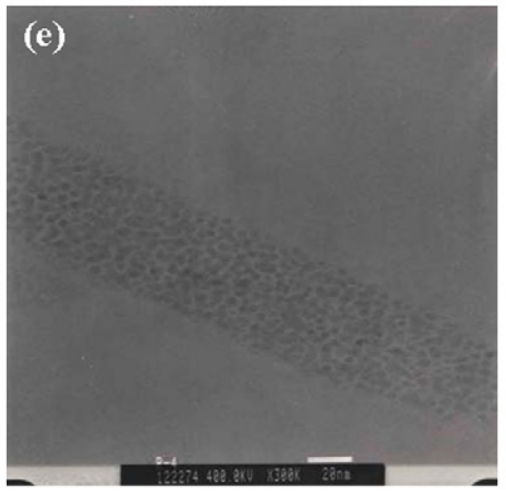

$\mathrm{pH} 7$

Fig. 2. Transmission election microscopic photographs of $\mathrm{TiO}_{2}$ powders obtained via the hydrothermal microemulsion process at (a) $\mathrm{pH} 4$, (b) $\mathrm{pH} 5$, (c) $\mathrm{pH}$ 6, (d) $\mathrm{pH} 7$, and (e) pH 7 (the magnified micrograph).

would occur during the hydrothermal reaction. The chloride ions connected to $\mathrm{Ti}^{4+}$ complex centers would be replaced by water to form $\mathrm{OH}_{2}{ }^{+}$ligands in the hydrothermal treatment. There should be two Ti-O-Ti bridges between two $\mathrm{Ti}^{4+}$ complex centers. Two or three octahedra would compose a primary particle through the above mechanisms in acidified starting microemulsion solutions. The following nucleation and crystal growth processes would cause the formation and crystallization of the spherical anatase-type titania particles. The particles observed in the TEM photographs were the secondary particles composed of the primary particles. With an increase in the $\mathrm{pH}$ value of the acidified microemulsion solution, the amount of $\left[\mathrm{Ti}(\mathrm{OH})_{3} \mathrm{Cl}_{3}\right]^{2-}$ would also increases, so that the linkage between two octahedra would become more probable leading to an increase in the number of primary particles. In turn, the size of the secondary particles would be larger.

On the other hand, the formation mechanisms of the rod-like particles prepared from the starting microemulsion solution at $\mathrm{pH} 7$ are quite different. As the $\mathrm{pH}$ value of the starting solution was high, the number of $\mathrm{OH}^{-}$groups joined with $\mathrm{Ti}^{4+}$ complex center was more. In this case, the number of $\mathrm{OH}^{-}$groups joined with $\mathrm{Ti}^{4+}$ complex center would be $4-5$. Increasing the number of $\mathrm{OH}^{-}$groups connected with $\mathrm{Ti}^{4+}$ complex centers would increase the probability of edge-shared bonding between two $\mathrm{Ti}^{4+}$ complex centers. The linkage of the two $\mathrm{Ti}^{4+}$ complex centers would be formed by dehydration of two $\mathrm{OH}^{-}$groups. The direction corresponding to the positions of the $\mathrm{Ti}^{4+}$ complex centers captured by $\mathrm{OH}^{-}$groups would extend continuously. 

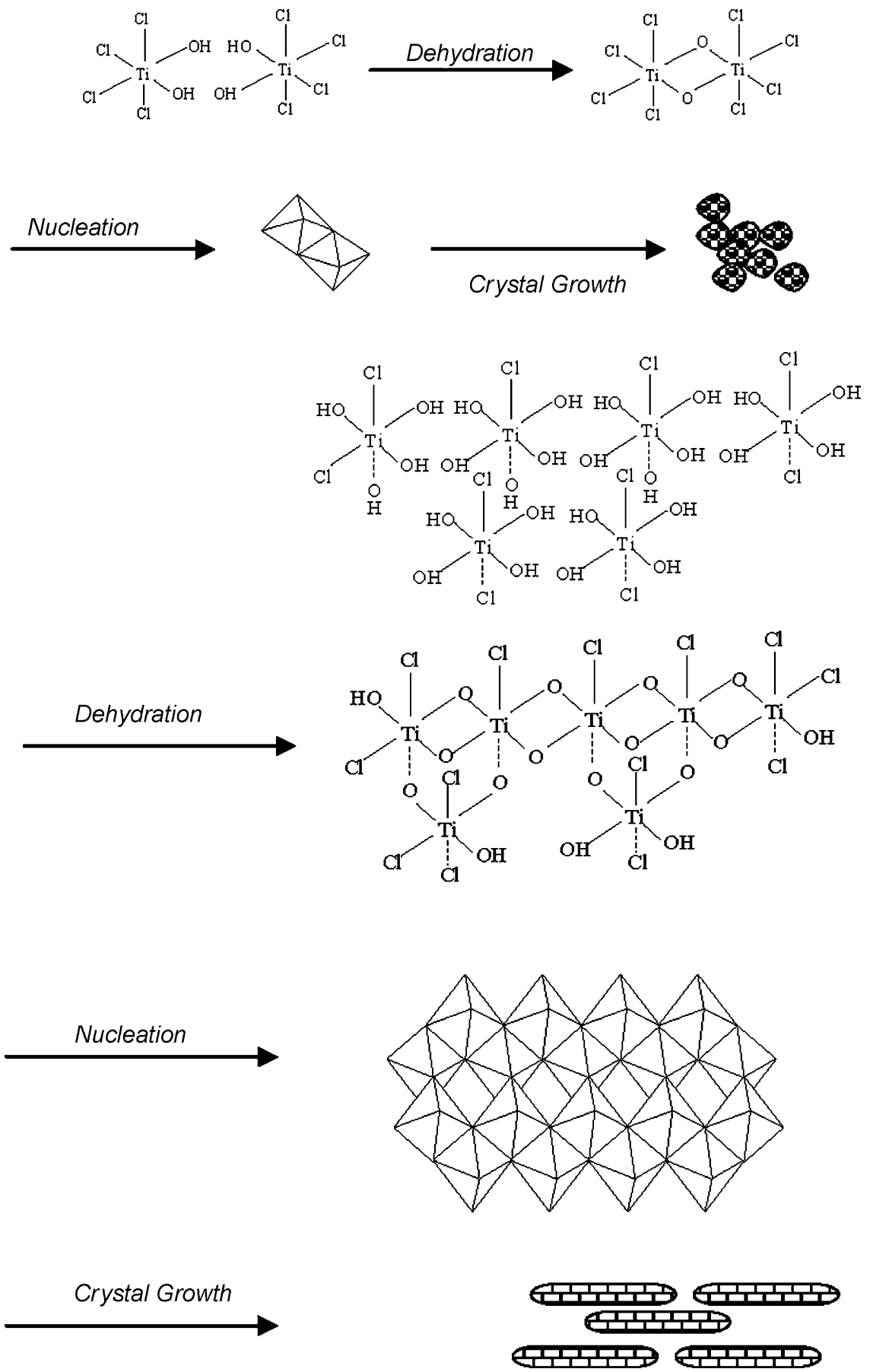

Fig. 3. Schematic diagram of the mechanisms for the formation of $\mathrm{TiO}_{2}$ powders in the hydrothermal microemulsion process.

The linkage of the octahedra would be lengthened in this direction by dehydration of four $\mathrm{OH}^{-}$groups. On the contrary, the linkage of octahedra would not expand in the direction corresponding to the positions of the $\mathrm{Ti}^{4+}$ complex centers occupied by chloride ions. Hence the growth of particle would be unidirectional. The resultant morphology of the primary particles is rod-like as explained by the above mechanisms. The water molecules would substitute the chloride ions connected to the $\mathrm{Ti}^{4+}$ complex center in the hydrothermal process. The secondary particles observed in the TEM photograph would be formed in the process of crystal growth.
3.2. Photocatalytic activity and surface area of $\mathrm{TiO}_{2}$ powders prepared via the hydrothermal microemulsion process

The photocatalytic activities of the obtained $\mathrm{TiO}_{2}$ powders were evaluated based on the decomposition of methylene blue. Fig. 4 illustrates the UV-vis spectra of methylene blue degraded by the powders synthesized at $\mathrm{pH} 4-7$ of the starting microemulsions. In all the spectra, the absorption intensity gradually decreased and remained their basic shape with an increase in UV illumination time. No intermediates [23] of methylene blue 


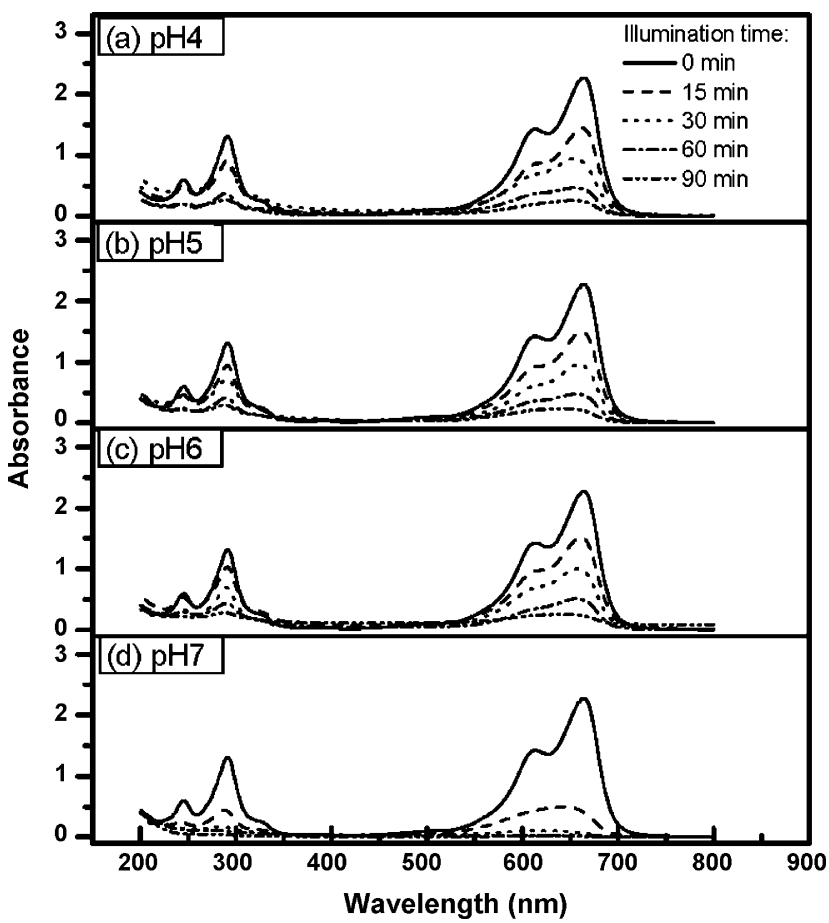

Fig. 4. UV-vis spectra of methylene blue degraded by $\mathrm{TiO}_{2}$ powders obtained via the hydrothermal microemulsion process at (a) $\mathrm{pH} 4$, (b) $\mathrm{pH} 5$, (c) $\mathrm{pH} \mathrm{6}$, and (d) pH 7 under UV light irradiation.

were found to exist during the photocatalytic process. Fig. 5 shows the variation of peak height of UV-vis absorbance at wavelength $=664 \mathrm{~nm}$ owing to the photocatalysis of $\mathrm{TiO}_{2}$ powders. The blank experiment was performed by using methylene blue solution without adding samples and irradiated for $90 \mathrm{~min}$ with an UV-lamp. Among all the samples, the rod-like powders

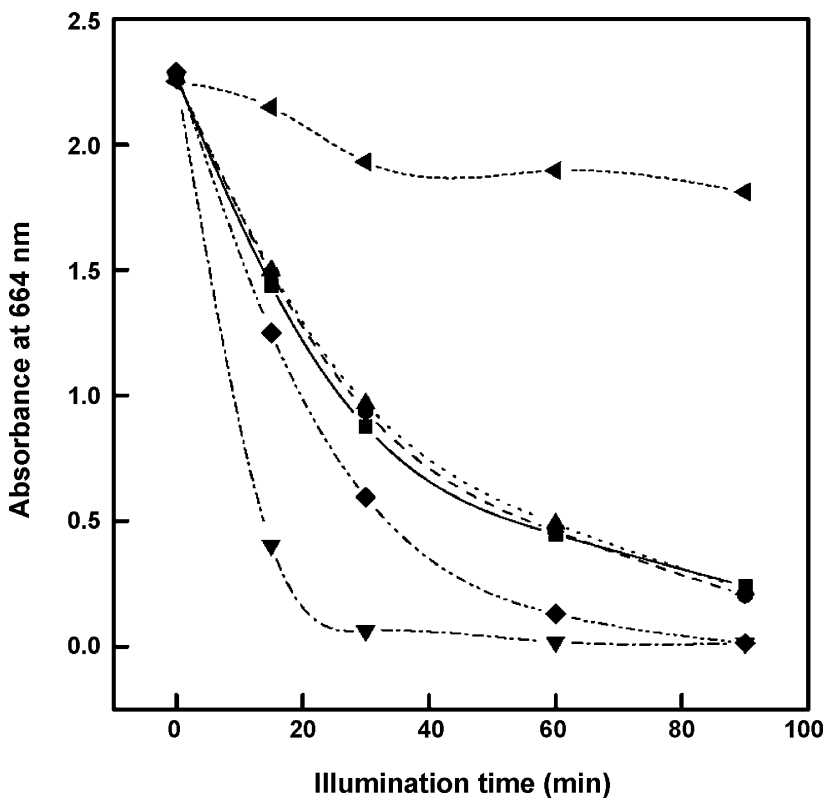

Fig. 5. Absorbance of UV-vis spectra at wavelength $=664 \mathrm{~nm}$ owing to the photocatalytic decomposition of methylene blue for samples prepared at (ם) $\mathrm{pH}$

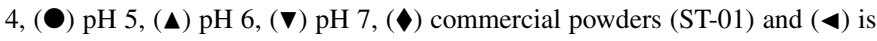
the blank test. prepared at $\mathrm{pH} 7$ possessed the highest photocatalytic activity which was even higher than that of the commercial powder ST-01 (Ishirara ST-01). On the other hand, the spherical particles synthesized at low $\mathrm{pH}$ conditions showed lower decomposition rates in comparison with the commercial ST-01 powder. The powders prepared at $\mathrm{pH} 4-6$ had similar low photocatalytic activities.

The specific surface areas of the prepared $\mathrm{TiO}_{2}$ powders were measured via BET. The surface areas of the spherical powders prepared at $\mathrm{pH} 4-6$ were similar, and the value was around $185 \mathrm{~m}^{2} / \mathrm{g}$. On the other hand, the rod-like powders prepared at $\mathrm{pH}$ $7 \mathrm{had}$ the largest surface area of around $260 \mathrm{~m}^{2} / \mathrm{g}$. From Fig. 2(e), it is observed that a large amount of pores were formed in the powders prepared at $\mathrm{pH} 7$. Due to pores formed in the particles and the rod-like morphology, the powders prepared at $\mathrm{pH} 7$ had a large BET surface area. The high photocatalytic activity of the rod-like powders prepared from neutral microemulsion was considered to result from their large surface area and the crystal planes exposed on the particle surface. The large surface area of the rod-like powders provided more area to react with methylene blue and also increased the probability to produce hydroxyl radicals and superoxide radical anions, thereby enhancing the photocatalytic activity. It was demonstrated that the developed hydrothermal microemulsion process successfully produced nanosized $\mathrm{TiO}_{2}$ powders with large surface area and high photocatalytic activity.

\section{Conclusions}

Nanosized $\mathrm{TiO}_{2}$ powders were successfully synthesized via the hydrothermal microemulsion process. Aqueous micelles in water-in-oil microemulsions were used as microreactors to confine the growth of $\mathrm{TiO}_{2}$ powders. The microemulsions were then hydrothermally heated at $150^{\circ} \mathrm{C}$ to produce ultrafine titania particles. The processing conditions had significant effects on the morphology and particle size of the formed powders. Low $\mathrm{pH}$ microemulsion solutions resulted in the formation of spherical particles. On the other hand, the neutral solution gave rise to rod-like particles. The mechanisms of nucleation and crystal growth of $\mathrm{TiO}_{2}$ particles were greatly affected by the acidity of the solutions. The rod-like particles had much larger specific surface area than the spherical particles; therefore, the rod-like particles exhibited significantly enhanced photocatalytic activity. The developed hydrothermal microemulsion process was demonstrated to prepare nanosized $\mathrm{TiO}_{2}$ powders with high surface area and improved photocatalytic activity.

\section{References}

[1] G.J. Wilson, A.S. Matijasevich, D.R.G. Mitchell, J.C. Schulz, G.D. Will, Langmuir 22 (2006) 2016.

[2] Z. Zhong, T.-P. Ang, J. Luo, H.-C. Gan, A. Gedanken, Chem. Mater. 17 (2005) 6814.

[3] C. Burda, X. Chen, R. Narayanan, M.A. El-Sayed, Chem. Rev. 105 (2005) 1025.

[4] K.C. Song, S.E. Pratsinis, J. Am. Ceram. Soc. 84 (2001) 92.

[5] R.J. Tayade, R.G. Kulkarni, R.V. Jasra, Ind. Eng. Chem. Res. 45 (2006) 922.

[6] S. Eiden-Assmann, J. Widoniak, G. Maret, Chem. Mater. 16 (2004) 6. 
[7] Q.H. Zhang, L. Gao, J.K. Guo, Nanostruct. Mater. 11 (1999) 1293

[8] Y. Murakami, T. Matsumoto, Y. Takasu, J. Phys. Chem. B 103 (1999) 1836.

[9] H. Cheng, J. Ma, Z. Zhao, L. Qi, Chem. Mater. 7 (1995) 663.

[10] J. Tang, S. Mei, M.F. Ferreira, J. Am. Ceram. Soc. 83 (2000) 1361.

[11] Q. Chen, Y. Qian, Z. Chen, G. Zhou, Y. Zhang, Mater. Lett. 22 (1995) 77.

[12] V. Chhabra, V. Pillai, B.K. Mishra, A. Morrone, D.O. Shah, Langmuir 11 (1995) 3307.

[13] E.J. Kim, S.H. Hahn, Mater. Lett. 49 (2001) 244.

[14] M. Lal, V. Chhabra, P. Ayyub, A. Maitra, J. Mater. Res. 13 (1998) 1249.

[15] M. Wu, J. Long, A. Huang, Y. Luo, Langmuir 15 (1999) 8822.

[16] M. Andersson, L. Osterlund, S. Ljungstrom, A. Palmqvist, J. Phys. Chem. B 106 (2002) 10674.
[17] P.D.I. Fletcher, A.M. Howe, B.H. Robinson, J. Chem. Soc. Faraday Trans. 83 (1987) 985.

[18] S. Somiya, R. Roy, Bull. Mater. Sci. 23 (2000) 453.

[19] Powder Diffraction File, Card No. 21-1272, Joint Committee on Powder Diffraction Standards, Swarthmore, PA.

[20] Cotton and Wilkinson, Advanced Inorganic Chemistry, 3rd ed., 1972, pp. 809-818.

[21] D. Nicholls, Complexes and First-row Transition Elements, 1st ed., 1974, pp. 139-148.

[22] U. Muller, Inorganic Structural Chemistry, John Wiley \& Sons, New York, 1993, pp. 45-46.

[23] T. Zhang, T. Oyama, A. Aoshima, H. Hidaka, J. Zhao, N. Serpone, J. Photochem. Photobiol. A 140 (2001) 163. 